\title{
Territorio y PRÁCticas CULTURAles amenazadas EN PUEblos yumanos en BAJA California
}

\section{Yuman Territories and Cultural Practices Under Threat in Baja California}

\section{Alejandra Navarro Smith ${ }^{1}$ Sergio Cruz Hernández ${ }^{2}$}

Resumen: En este artículo reflexionamos sobre la relación entre prácticas culturales, identidad, y las ideas de bienestar que surgen desde las voces de los miembros de dos pueblos yumanos: cucapá y pa'ipai. En ambos casos convergen factores que dificultan el acceso a sus territorios donde realizan las prácticas culturales de pesca y recolección de piñón, respectivamente. En sendos ejemplos, los autores identifican la continuidad de procesos de despojo territorial, con argumentos de desarrollo, conservación o deshabitación del territorio; sin embargo, las lógicas que subyacen son en realidad dinámicas sociales de producción de desigualdad basadas en criterios que estigmatizan las prácticas de subsistencia propias de estos pueblos yumanos. Como veremos a lo largo del documento, los cucapá y los pa’ipai viven un proceso de

1 Doctora en Antropología Social por la Universidad de Manchester. Líder del CA Indígenas y Globalización. Sus líneas de especialización incluyen: conflictos socioambientales y judiciabilidad de derechos de pueblos y comunidades indígenas; y Etnicidad, representación y relaciones interétnicas en situaciones de conflicto. Obtuvo una cátedra de Conacyt en CIESAS-Occidente.

Correo electrónico: alenavarrosmith@yahoo.com

2 Doctor en Ciencias y Humanidades para el Desarrollo Interdisciplinario por la Universidad de Coahuila. Profesor de tiempo completo de la Facultad de Ciencias Administrativas y Sociales-UABC. Sus líneas de especialización son: representaciones sociales de la relación con la naturaleza, Identidades sociales y Cibercultura. Actualmente se desempeńa como subdirector de la Facultad de Ciencias Humanas-UABC.

Correo electrónico: checoch@uabc.edu.mx

Fecha de recepción: 2807 15; Fecha de aceptación: 061115.

(cc) EY-NC-ND Páginas 75-102. 
etnización, en donde la lucha por acceder a sus territorios históricos está en el centro de sus procesos de recreación identitaria, reivindación étnica y participación política.

Palabras clave: prácticas de recolección y pesca, cucapá, pa’ipai, despojo territorial, formación de sujeto político colectivo (etnización)

Abstract: In this article we look at the intersections between identity and the ideas of wellbeing that emerge from current gathering and fishing practices amongst contemporary Yuman people. We analize how the menace of dispossession over Cocopah and Pa'ipai territories plays a role in reproducing the inequalities, when their right to continue these cultural practices is impeeded by the force of environmental laws or private property. As we discuss, these contemporary Yuman people are experiencing a process of ethnification whereby their struggle to remain in their historic territories is at the centre of their identities as contemporary Cocopah or Pa'ipai and guides their political participation.

Keywords: Gathering and fishing, Cocopah, Pa'ipai, territorial dispossession, ethnification

\section{Introducción}

Los pueblos yumanos — cucapá, pa'ipai, kumiai y kiliwa—, ubicados en Baja California (ver mapa 1), comparten preocupaciones por amenazas o acciones que derivan en el despojo de sus territorios; también expresan un interés generalizado por la revitalización y reproducción de sus culturas en un momento en que, por ejemplo, la lengua cucapá está clasificada como en alto riesgo de desaparición en el Atlas de la Unesco (Moseley, 2010). Es en este contexto, en el que pueblos y comunidades indígenas están retomando los tratados internacionales en materia de derechos indígenas para mirar hacia adentro de sus propias formas de organización y reconstituirse como pueblos originarios. 
Mapa 1. Ubicación de los pueblos yumanos.

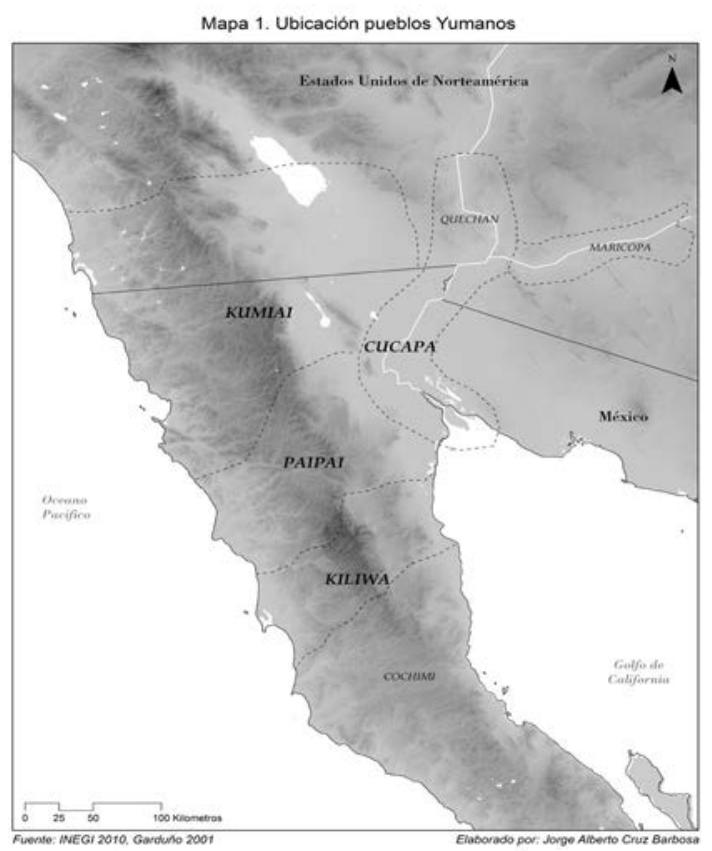

En este entrecruzamiento de la historia de despojo y asimilación cultural, transformación de sus identidades, procesos de formación de un sujeto político colectivo ${ }^{3}$ y un presente que añade un conjunto de leyes y acuerdos internacionales que les reconocen derechos, es que los pueblos y comunidades indígenas se organizan para hacer frente a las circunstancias que los siguen amenazando. Sus subjetividades de pueblos indígenas se están transformando en relación con este contexto de interacción con organizaciones de la sociedad civil, académicos, instancias de gobierno; en particular en su interacción con estas últimas - $y$ un compendio de marcos legales que limitan su acceso al territorio de

\footnotetext{
${ }^{3}$ Siguiendo a Restrepo, entendemos como etnización: "el proceso de formación de un sujeto político en un sentido amplio - un nosotros/ellos-, y de unas subjetividades —unas identificaciones - en nombre de la existencia — supuesta o efectiva- de un 'grupo étnico'” (2011: 40). En este caso se trata de la formación de un sujeto político colectivo, los pueblos yumanos en general, y cada grupo étnico en particular con los referentes de sus propias luchas.
} 
convivencia en un entorno natural, sociocultural, político y económico- es que vemos acentuadas las desigualdades. En este entorno de amenazas por las dinámicas del "desarrollo global" es que los pueblos originarios se movilizan para defender la reproducción de sus culturas, en este caso a través de las prácticas de recolección y de pesca, mismas que muchas veces son sus más importantes medios de subsistencia.

En este artículo reflexionamos sobre la relación entre prácticas culturales, identidad y las ideas de bienestar que surgen desde las voces de los miembros de dos pueblos yumanos: cucapá y pa’ipai. En ambos casos convergen factores que dificultan el acceso a sus territorios donde realizan las prácticas culturales de pesca y recolección de piñón, respectivamente. En sendos ejemplos, los autores identifican la continuidad de procesos de despojo territorial con argumentos de desarrollo, conservación o deshabitación del territorio; sin embargo, las lógicas que subyacen son en realidad dinámicas sociales de producción de desigualdad basadas en criterios que estigmatizan las prácticas de subsistencia propias de los pueblos yumanos. Como veremos a lo largo del documento, los cucapá y los pa’ipai viven un proceso de formación de un sujeto político colectivo - proceso de etnización en palabras de Restrepo (2011)—, en donde la lucha por acceder a sus territorios históricos está al centro de sus procesos de recreación identitaria, reivindicación de derechos como pueblos indígenas y participación política.

En términos más amplios, estos casos nos permiten entender relaciones entre 1) acciones de autoridades derivadas de programas de políticas públicas diseñadas sin tomar en cuenta la especificidad de las poblaciones indígenas, y 2) la importancia de los territorios para la reproducción de ciertas formas de vida propias de las culturas yumanas. ${ }^{4}$

\footnotetext{
${ }^{4}$ Las culturas yumanas se han documentado por la arqueología, la historia, la lingüística y la etnografía. Para el caso cucapá, los estudios académicos que reiteran que el Delta del Río Colorado ha sido territorio históricamente habitado por los cucapá incluyen los arqueológicos (Ortega 2004; Porcayo y Rojas, 2009 y 2010; Porcayo et al., en prensa); reportes de restos arqueofaunísticos (Porcayo y Guía, 2007 y 2008), históricos (Gómez, 2000) y etnográficos (Gifford, 1933; Kelly, 1973; Álvarez, 2004). Para los pa'ipai ver los estudios realizados por Hohental, (2001) en los que se sistematizan los ejes de materiales líticos, etnografía de la región, subsistencia y cultura material pa’ipai, y de otros pueblos yumanos. En muchos casos, las culturas indígenas se estudian como si fueran un asunto del pasado, o desde un enfoque que tiende a esencializar algunos
} 
Lo que observamos es la disminución del acceso al territorio que viene acompañado de precarización en la calidad de vida, como más adelante mostramos con los ejemplos que se presentan. Como respuesta ante los diversos tipos de obstáculo para acceder a sus territorios — sea la instalación de cercos que delimitan propiedades privadas o la creación de áreas naturales protegidas-, estos pueblos yumanos se organizan para que el respeto de sus territorios sea entendido como una obligación del Estado.

El problema al que se enfrentan los pueblos originarios es claro: por una parte, las autoridades intentan proyectar una relación de respeto y cumplimiento de los derechos demandados al reunirse con ellos para negociar (Navarro y Bravo, manuscrito); pero, por otro lado, promueven la incorporación de estos grupos a las dinámicas reguladas por las lógicas del capital —al privatizar su territorio o al legalizar la pesca cucapá mediante la asignación de permisos de pesca, por ejemplo (Navarro, 2008a)_. Incluso a nivel federal, la llamada "reforma energética" también es una continuidad de la dinámica de asimilación y despojo que se actualiza al supeditar el derecho de los pueblos a su territorio a la lógica del capital global. Como lo señalaron un grupo de mujeres indígenas en la Conferencia Mundial de Pueblos Indígenas (CMPI) en Nueva York, octubre de 2014.

Esta reforma energética es la punta de lanza para el despojo, la criminalización, la ocupación y el genocidio de los pueblos que aún permanecemos en nuestros territorios, y por estar cuidando nuestros territorios aún conservamos los recursos, que ellos ven como recursos y nosotros los vemos como bienes naturales de los que vivimos - Leyes secundarias de la Reforma energética- (Carlsen, 2014)

de sus rasgos culturales. Recientemente se han publicado textos que ayudan a entender que, si bien las culturas yumanas tienen orígenes históricos, es decir, de antes de la llegada de colonizadores no indígenas, también están en constante transformación. Y que dichas transformaciones son las expresiones culturales de pueblos indígenas del siglo XXI (Muelhmann, 2013; Navarro 2012). 
En efecto, el doble discurso que denunciaron las lideresas en la CMPI está claro incluso en el documento titulado Uso de tierras, sostenibilidad $y$ derechos humanos en el sector energético ${ }^{5}$ de la Secretaría de Energía (Sener): primero se describen todos los medios para hacer respetar los procesos de consulta previa, libre e informada, retomando los convenios en materia de derechos de pueblos y comunidades indígenas que México ha ratificado; al terminar esa sección se indica en los mecanismos para regular la contraprestación por el uso de tierras:

Las actividades de exploración y extracción de hidrocarburos se consideran de interés social y orden público, por lo que tendrán preferencia sobre cualquier otra que implique el aprovechamiento de la superficie y del subsuelo de los terrenos afectos a aquellas. La legislación secundaria establece el mecanismo basado en principios de transparencia y equidad para definir los términos y las condiciones generales de la contraprestación, los términos y las condiciones para el uso, goce o afectación de los terrenos, bienes o derechos necesarios para realizar las actividades (íbid, el resaltado es nuestro).

Así se establece la prioridad de "exploración y extracción" sobre cualquier otro uso de la tierra, es decir, los usos que les puedan dar los pueblos y comunidades cuyos proyectos de vida se desarrollan en estrecha relación con ellos. La reforma energética, al incorporar la cláusula de "ocupación temporal" le da todas las atribuciones a los agentes del Estado y el mandato legal para efectivamente expropiar terrenos bajo este principio de prioridades.

Ésta es la lógica de la histórica relación entre Estado y pueblos indígenas en México. La reforma energética, en particular, intenta proyectar que existen una serie de medidas compensatorias y justas para el uso del territorio de los pueblos y comunidades indígenas con fines de exploración y extracción de hidrocarburos. La realidad es que si los

\footnotetext{
${ }^{5}$ Leyes secundarias de la reforma energética [en línea] disponible en http://www.energia.gob.mx/webSener/leyes_Secundarias/9220.html [fecha de consulta: 18 de junio de 2015].
} 
pueblos deciden no vender, no estarán en posibilidad de que esta decisión sea respetada. La no-venta se construye contraria al argumento del desarrollo de la nación y la atracción de capitales, reproduciendo así el estereotipo del retraso económico y cultural de las comunidades y pueblos indígenas. Es por ello que, comparando este proceso con el caso del despojo que del territorio de los pueblos yumanos se ha venido dando, decimos que esta forma de expropiación — disfrazada de compra- es un ejemplo de la continuidad de las prácticas de desposesión de los territorios históricos y ancestrales de los pueblos indígenas.

Según Alejandro Vázquez, en los procesos de organización y defensa de los pueblos indígenas contemporáneos en México,

la desigualdad [debe ser] una plataforma analítica que convoca a ligar a la modernidad, la globalidad y las relaciones de poder como procesos inevitables para entender los dilemas y encrucijadas de aquellas sociedades y culturas propias del sur globalizado, tal y como lo han descrito Catherine Walsh, Arturo Escobar y Walter Mignolo. Frente a la reinvención de los grandes paradigmas del mundo moderno: el desarrollo, la igualdad y el progreso, emergen también nuevas formas locales de organización y defensa de sus particularidades culturales y naturales, revitalizando identidades, recreando saberes y adaptando elementos de una memoria ancestral que funciona para hacer frente a los procesos del Estado y el mercado global (Vázquez y Terven, 2015: 1).

Es en este tenor que a continuación describimos los esfuerzos de los pueblos cucapá y pa'ipai para seguir realizando prácticas culturalmente significativas en el contexto de los procesos de reconstitución de sus identidades étnicas en el entorno de exclusión cultural, social y económica en el que actualmente viven en Baja California. Como estrategia de exposición, primero presentamos la información estadística y datos generales de los pueblos cucapá y pa'ipai: nombres, número de personas que componen esos grupos étnicos, lugares donde viven, patrones de asentamiento e información estadística que permita caracterizarlos. En 
el segundo apartado se describen las prácticas culturales amenazadas de estos pueblos, asociadas a la historia del despojo de sus territorios históricos en Baja California; en este segundo apartado daremos cuenta de la importancia del territorio y los principales problemas que enfrentan los pa'ipai de Santa Catarina para mantener la práctica de la colecta del pińón (Cruz, 2015), y los problemas que enfrentan los cucapá para seguir pescando en el Delta del Río Colorado (Navarro 2008a; Navarro 2010; Navarro, Bravo y López, 2013; Navarro, Bravo y López, 2014). Finalmente, nos aproximamos a los procesos de reconstitución y configuración actual de los pueblos cucapá y pảipai a partir de los procesos descritos. Aquí incorporamos aspectos socioculturales, simbólicos, políticos y económicos implicados en la reconfiguración de la relación entre los pueblos yumanos y la sociedad bajacaliforniana; y proponemos cómo estos aspectos están asociados a las continuidades/discontinuidades/transformaciones de sus prácticas culturales, la defensa de su territorio y los procesos de negociación de su reconocimiento como poblaciones originarias con derechos dentro del Estado mexicano.

\section{Estadísticas e indicadores sobre cucapás y pa’ipais}

Los cucapá y los pa' ipai son dos de los cuatro pueblos yumanos que actualmente se hacen presentes en el espacio público/político bajacaliforniano para demandar a las autoridades la solución de conflictos que afectan sus formas de vida o amenazan con despojarlos de su territorio. ${ }^{6}$ Estos pueblos originarios del noroeste mexicano y suroeste estadounidense han sido afectados por las políticas públicas nacionales e internacionales que han trazado límites administrativos sobre el territorio histórico y ancestral de dichos grupos étnicos. Para efectos de claridad en la exposición, se revisarán las características actuales y los procesos de colonización con el fin de develar las relaciones de poder, los intereses y ciertos patrones de relaciones sociales entre los pueblos originarios y otros pobladores, modelados en este contexto particular.

Según Garduño, "los asentamientos yumanos más poblados son los de Santa Catarina (pa'ipai-kwatl) y la Huerta (k'miai-cochimíe) con apenas 169 y 142 habitantes, respectivamente, seguidos por San Isidoro

\footnotetext{
${ }^{6}$ Los otros dos pueblos originarios de Baja California son kumiai y kiliwa.
} 
(pa'ipai) y San José de la Zorra (k'miai), con 77 habitantes cada uno, y por último Arroyo de León (kiliwa) con 33 indígenas. En ocho ejidos indígenas viven 700 personas" (Garduño, 1995, citado en Garduño 2009: 22). ${ }^{7}$ Para el caso de los cucapá, el Censo de Población y Vivienda 2010 indica la presencia de 145 cucapás en México, identificados en el censo por hablar una lengua indígena. De ellos 78 se ubican en el municipio de Mexicali, 43 en San Luis Río Colorado en Sonora, 11 en Ensenada, 2 en Tecate, 2 en Jalisco y 1 respectivamente en Tijuana, el Distrito Federal, Guerrero y Nayarit (Inegi, 2010). La CDI por su parte, contabilizó 344 cucapás sólo en Sonora y Baja California en un conteo que realizó en 2005.

Como se puede notar, al comparar sólo estos dos conteos, el número de miembros del pueblo cucapá incrementa si el indicador es el de autoidentificación y no sólo por hablar la lengua. Además, se localizan cucapás en Somerton, Arizona, y en otros estados de la unión americana. Para agregar complejidad a la forma de contabilizar y ubicar a los cucapá, según información proporcionada por el Instituto Nacional de Lenguas Indígenas (Inali), éstos se ubican también en ejidos del valle de Mexicali como el ejido Alberto Oviedo Mota (El Indiviso), en la colonia La Puerta, en el ejido Cucapá Mestizo, en el ejido Durango, en el ejido México, en el ejido Sonora, en los campos Flores, Sonora, Camerina, El Prado, e incluso, muchos de ellos, radican y trabajan en Mexicali. Con estas estadísticas e información se puede inducir que la dispersión es una característica del patrón de asentamiento de los cucapá en la actualidad, aunque oficialmente les sean reconocidos sólo tres poblados de uno y otro lado de la frontera: el Mayor Cucapah (sic) en Baja California, Pozas de Arvizu en Sonora, y Somerton en Arizona.

\footnotetext{
${ }^{7}$ Ahora, según el Censo de Población y Vivienda 2010, Santa Catarina está habitado por 133 personas en 27 viviendas. Aunque de acuerdo con verificación de campo realizada por López y Ovalle en noviembre de 2011, se apunta que existen diferencias ante esta información: “...pues de acuerdo a los datos recabados mediante la aplicación de una cartografía y un cuestionario, la comunidad cuenta con 58 casas, de las que 23 están habitadas y 35 están en situación de abandono, debido a la migración a poblados y ciudades vecinas, así como a Estados Unidos. Un fenómeno que también forma parte de los procesos de globalización. La población total es de 90 habitantes; 43 mujeres y 47 hombres [en Santa Catarina]".
} 
En cuanto a la información sobre la situación económica de estos pueblos, Garduño (2009: 105) observa que entre los yumanos, 35\% de la población económicamente activa (398 personas) trabaja contratada regularmente a lo largo del año; $21 \%$ lo hace menos de 4 días por semana; y $22 \%$ menos de 5 horas diarias. El ingreso promedio de estos trabajadores yumanos es de 200 dólares (US) por mes. Según la misma fuente, los grupos yumanos están considerados oficialmente como población que vive en condiciones de extrema pobreza (ibídem). Este tipo de estadísticas apunta entonces a la precariedad del empleo y a los ingresos reportados en el límite de los 6.6 dólares diarios, que aplica sólo para ese $35 \%$ reportado como "población yumana que trabaja". El resto, es decir, 65\%, no es captado por esta forma de producir información sobre la situación económica de los pueblos originarios.

\section{Patrón de asentamiento disperso y factores de asimilación. Reflexiones desde el caso cucapá}

Los cucapá han enfrentado un proceso de asimilación a las dinámicas de vida, territoriales y del aprovechamiento de los recursos naturales impulsadas por las políticas públicas en el Delta del Río Colorado desde la compra de su territorio en 1886. A diferencia de los pueblos mesoamericanos, la evidencia arqueológica señala que los pueblos del norte de México tenían patrones de asentamientos dispersos (Porcayo et al., en prensa); y según las estadísticas presentadas con anterioridad, esto parece seguir siendo así. Sin embargo, los procesos de desarrollo que han hecho del Delta del Río Colorado un redituable territorio de producción de hortalizas para la exportación pueden ser leídos en la clave de despojo del territorio histórico del pueblo cucapá, e incluso, la asimilación de las formas de organización y de cultura cucapá que observaron Gifford (1933) y Kelly (1973) en esas dinámicas del desarrollo impulsadas por las instituciones del Estado mexicano.

Entre 1930 y 1970, los cucapá pasaron de ser un pueblo indígena con patrones culturales, formas de organización y territorios propios, como los describieron Gifford (1933) y Kelly (1973), a una "situación crítica”, tal y como caracteriza al cucapá el Atlas interactivo de la Unesco de lenguas en peligro en el mundo (Moseley, 2010). ¿Qué factores 
influyeron para que el proceso de asimilación a las formas culturales y lengua dominante ocurrieran en tan poco tiempo? El patrón de asentamiento disperso podría ser una explicación; los cambios en el ecosistema deltáico, otra causa (Gómez, 2000: 149-50; Porcayo et al., en prensa); los programas "de desarrollo" impulsados por políticas públicas podrían ser una razón más. En combinación, los tres factores anteriores podrían constituir una matriz multifactorial compleja que expliquen esta transformación acelerada de la cultura cucapá.

Para ejemplificar el tipo de cambios ocurridos en interacción con ciertas acciones de agentes del Estado se puede tomar el caso de la fundación del asentamiento conocido como El Mayor Cucapah (sic). A finales de los 70, el exceso de lluvia en Colorado provocó la crecida del río, y con ello la liberación de cuotas adicionales de agua hacia México. El agua provocó una inundación en la región donde vivían dispersos los cucapá en las orillas de los ríos Colorado y Hardy, entre lo que actualmente sería San Luis Río Colorado, el Valle de Mexicali y hasta la Sierra Cucapá. En el mapa 2 que retomamos del Atlas de Riesgos elaborado por el gobierno de Baja California (ver mapa 2, en página siguiente.)se aprecia con claridad la zona del Río Hardy —indicada en el recuadro sombreado-, área de residencia de un grupo cucapá que fue inundada en el periodo referido. 
Mapa 2. Las zonas inundadas están dentro del recuadro sombreado.

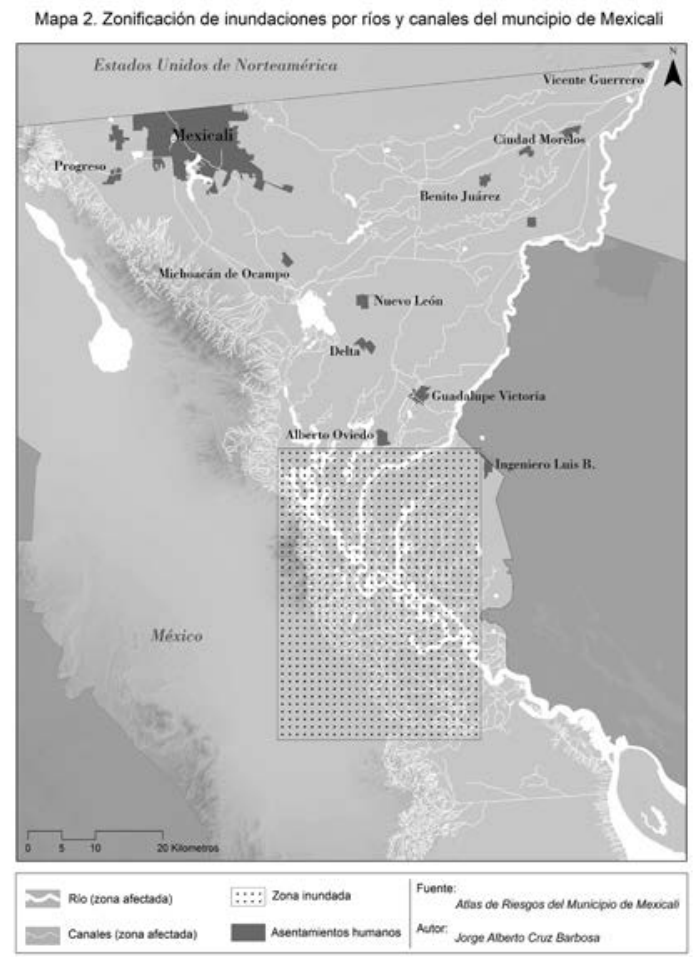

Ante la emergencia, el gobierno de Mexicali asignó a los cucapá un terreno más alto y les proveyó "pies de casa", esto es, un terreno de 10x10 metros, bloques de concreto y láminas de asbesto. Fue así como a principios de los ochenta se inició la construcción del poblado oficial cucapá en Baja California. ${ }^{8}$ En este caso concreto lo que se observa es

\footnotetext{
${ }^{8}$ Los cucapás que no viven en el Mayor, posiblemente la mayoría, están fuera de la estructura de oportunidades que se ofertan para los pueblos indígenas, pues es requisito demostrar la residencia en la "comunidad oficialmente reconocida" como cucapá, en este caso. Esta tendencia del Estado mexicano de identificar un solo centro de población para cada grupo indígena apunta a una relación ADMINISTRATIVA que ignora la diversidad cultural de los pueblos originarios en el país, y que para efectos da facilidad a su propio aparato burocrático, pues sólo reconoce a quienes habitan en el Mayor Cucapah (sic) como beneficiarios de sus programas. Se podría decir que la definición de "comunidad indígena mesoamericana" es la que ha modelado la forma
} 
la transformación de un patrón de asentamiento —en el que se ubicaba una familia muy alejada de la otra- a la creación de un poblado con predios uno al lado del otro. También se observa un cambio en los materiales con los que se construían los espacios para habitar. En entrevistas, los cucapá relatan que todavía en los años setenta sólo se construían refugios, y no casas de bloque con techo de lámina: se veía "una señora sola por allá, debajo de una 'ramada' hecha con cachanilla, bajo un mezquite. Otra con sus hijos más allá. De noche los hombres las visitaban, pero muchas no vivían con ellos. Sólo ellas y sus hijos" (entrevista en archivo).

Desde entonces, los cucapá que han vivido en El Mayor Cucapah (sic) relatan con frecuencia su preferencia por vivir fuera de ahí, e incluso quienes lo hicieron de manera temporal o permanente. Doña Inocencia Sáiz, por ejemplo, dejó temporalmente el poblado cuando tuvo la oportunidad de establecerse junto con sus hijos menores a la orilla de la Laguna Salada, a donde desviaron el excedente de agua de las inundaciones referidas. El agua rellenó la antigua laguna seca que se llenó de carpas y camarones. Doña Inocencia Sáiz se llevó a sus hijos a vivir con ella en campamentos improvisados al pie de la Sierra Cucapá frente a la laguna. "Allá vivimos mucho tiempo, como diez años. Así, a la intemperie. Así le gustaba vivir a mi mamá”, recuerda Toña, la hija de Inocencia que compartió con ella esta experiencia.

Otros cucapá que tuvieron la posibilidad de salir de El Mayor lo hicieron a la primera oportunidad. Algunos se fueron para alejarse de los conflictos internos derivados de disputas por dinero al manejar proyectos productivos, o generados por discusiones en la asamblea de bienes comunales. Algunos se fueron para ofrecer a sus hijos diferentes horizontes de educación o buscar mejores oportunidades de empleo en ciudades como Mexicali, Ensenada o incluso en Estados Unidos (Montaño, 2015). Don Rosario prefirió salir de El Mayor para residir con su mujer al pie de la sierra. En relativo aislamiento, don Chayo vivió hasta sus últimos días a la manera que lo hicieron sus ancestros.

Uno de los procesos recientes de asimilación que ha incidido en la forma de organización de los cucapá ha sido el que han generado las

de definir a los pueblos indígenas en México. 
políticas de pesca y de protección al medio ambiente en el Delta del Río Colorado (Navarro, 2008a). El marco legal de pesca y de medio ambiente se desprende de políticas públicas que no reconocen los derechos diferenciados de los pueblos indígenas (Navarro, Bravo y López, 2013). De este modo, al exigirles a los cucapá la observación de este tipo de leyes se les continúan imponiendo formas de organización y formas de vida modeladas por proyectos de desarrollo que han afectado gravemente su acceso al territorio, obligándolos a adaptarse a las nuevas propuestas aun a costa de la transformación de sus instituciones culturales.

Por último, con la creación de la Reserva de la Biósfera del Alto Golfo de California y Delta del Río Colorado los cucapá que todavía pescan han visto impedida esta actividad en la zona núcleo de la reserva, que coincide con la desembocadura del río en el mar, donde ellos tienen sus campamentos de pesqueros. Algunos cucapá se han resistido a perder el acceso a esta zona donde todavía pueden pescar, y por ello han tenido que pagar el alto precio de la criminalización por defender esta parte de su territorio y de sus actividades culturalmente significativas, desafiando el marco legal vigente en la zona (Navarro, 2010). En este escenario de acoso, los cucapá organizados en la Sociedad Cooperativa Pueblo Indígena Cucapá Chapay Seisjhiurrar (SCPICCS), con el apoyo de abogados solidarios ${ }^{9}$ y un equipo de asesores interdisciplinarios, ${ }^{10}$ judicializan sus derechos para hacer notar a los tomadores de decisión que ciertas normas — en materia de medio ambiente y de pesca - son violatorias de los derechos diferenciados que la Constitución mexicana les reconoce como miembros de un pueblo

\footnotetext{
${ }^{9}$ En 2014, el abogado responsable del acto de defensa que en este documento se estudia fue el Lic. Daniel Solorio Ramírez, apoyado por otros abogados solidarios, entre los que se encuentran la Lic. Yacotzin Bravo, adscrita a la UNAM; el Lic. Ricardo Rivera de la Torre, de la UABC; y los licenciados Marcos de la Cruz y Eucadio Macías García, litigantes independientes.

${ }^{10}$ Este equipo interdisciplinario suma a los abogados a Raúl Ramírez Baena, presidente de la Comisión Ciudadana de Derechos Humanos del Noroeste (CCDHN) y exombudsman de la Procuraduría de Derechos Humanos de Baja California; a la Lic. Lorena Rosas, defensora de derechos humanos, periodista y miembro de la CCDHN; a la Biol. Catalina López-Sagástegui, adscrita a Ucmexus de la Universidad de California Riverside, y a la antropóloga, la primera autora de este documento, en ese momento adscrita al Instituto de Investigaciones Culturales-Museo y actualmente catedrática de Conacyt en CIESAS-Occidente.
} 
originario. El caso ejemplifica las dificultades a las que se enfrentan los pueblos nativos cuando se ven obligados a recurrir al sistema judicial para demandar la protección de derechos. La judicialización de los derechos de los pueblos y comunidades indígenas (Sieder, Schjolden y Angell, 2011; Gálvez, 2013) es un fenómeno que se ha documentado ampliamente y que se explica a partir de la creación y difusión de marcos internacionales que reconocen los derechos de poblaciones originarias en naciones independientes.

Desafortunadamente, para el caso cucapá se ha documentado cómo las respuestas de los tomadores de decisión están modeladas por el conjunto de procedimientos, valoraciones y prejuicios que pesan sobre los indígenas en la cultura jurídica central: les responden de manera confusa, reafirmando el orden de las cosas, el mismo orden en el que se genera la violación del derecho para el que le piden protección o para el que se demanda un cambio incluso en la forma de pensar de los tomadores de decisión (Navarro 2014; Navarro, Bravo y López, 2013 y 2014). Uno de los ejemplos es el amparo que los cucapá organizados en dos cooperativas solicitaron en 2014 contra el "tope de captura". En su resolución, la jueza ignora los argumentos que se le ofrecen y que le permitirían actuar conforme a la ley para otorgarle a los cucapá de ambas cooperativas la suspensión provisional contra el tope de captura; ello en el entendido de que los cucapás, como habitantes históricos y presentes del Delta del Río Colorado, estarían siendo privados de su derecho territorial como pueblo indígena y al derecho preferente de las comunidades al uso y disfrute de los recursos naturales de los lugares que ocupan. ${ }^{11}$

${ }^{11}$ En otro lugar hemos documentado que "[s]obre estos derechos existe una tesis contenida en el amparo en revisión 123/2002. Comunidad indígena de Zirahuén, Municipio de Salvador Escalante, Michoacán. 4 de octubre de 2002. Cinco votos a favor de los resolutivos; mayoría de tres votos en relación con las consideraciones. Disidentes: Juan Días Romero y Guillermo I. Ortiz Mayagoitia. Ponente: Mariano Azuela Güitrón. Secretaria: Lourdes Ferrer Mac Gregor Poisot...” Novena Época. Instancia: Segunda Sala. Fuente: Semanario Judicial de la Federación y su Gaceta. Tomo: XVI, Noviembre de 2002. Página: 445. Tesis: 2a. CXXXVIII/2002, fuente retomada de la "queja urgente" enviada por el Lic. Daniel Solorio al Juez $1^{\circ}$ de Distrito del Estado de Baja California con relación a la resolución provisional del amparo 180/2014-4” (Navarro, Bravo y López-Sagástegui, 2014). 


\section{Transformaciones de la identidad entre los pa'ipai de Santa Catarina: asentamiento y la recolección del piñón}

Como en el caso de los cucapá, los pảipai mantienen un patrón de asentamiento disperso, aunque se les reconoce un solo asentamiento oficial, el de Santa Catarina. En otro lugar ya se ha documentado que,

[...] los pa'ipai constituyen parte del segmento yumano de los indígenas serreños. Esto se debe a que el lugar donde actualmente habitan es la sierra de Juárez. Pese a esto, los pa'ipai se reconocen a sí mismos como gente del desierto, debido a que su procedencia se ubica en el desierto de Arizona, incluyendo las tierras interiores del Gran Cañón, en donde todavía viven algunos grupos que son miembros de la familia pai: los yavapai, wala pai y havasupai. De esa árida región, los pa'ipai llegaron a Baja California hace 600 años, razón por la cual son considerados como el grupo de más reciente arribo ... En la actualidad los pa'ipai son ubicados como residentes de una sola comunidad, la ex-misión de Santa Catalina (establecida en 1797), comunidad denominada como Santa Catarina. Este lugar se localiza a 120 kilómetros al sur de la línea fronteriza con Estados Unidos, exactamente al centro de la península de Baja California: 80 kilómetros al oeste del Océano Pacífico e igual distancia al este del delta del río Colorado, en el golfo de California. En esta población viven cerca de 300 indígenas, aunque anteriormente numerosas familias pa'ipai habitaban en diversos lugares, mismos que fueron abandonando por la falta de agua, la presión mestiza sobre su tierra e incluso como resultado del despojo de su ancestral territorio (Garduño, 2013: 500). 
En Santa Catarina, de acuerdo con el testimonio de Teresa Castro, ${ }^{12}$ habitan indígenas que hablan distintas lenguas, aunque comúnmente se la conoce como una comunidad pa'ipai en realidad hay habitantes pa'ipai, otros ko'atl y otros más de descendencia kiliwa. La señora Teresa habla tanto el pa'ipai como el ko'atl, aunque en otras familias sólo se habla una u otra lengua.

La recolección del piñón es un elemento central de la adaptación de estos grupos al medio en el que viven; de su carácter seminómada estacional se desprende la costumbre de acudir durante el verano al bosque piñonero (Pinus quadrifolia) en la sierra de Juárez y a las costas del Océano Pacífico a recolectar almeja, mejillón o abulón y pescar durante el invierno. Como veremos más adelante, más allá del carácter de sobrevivencia por el seminomadismo, las formas sociales de organización aparejadas a la recolección del piñón tienen una importancia fundamental para entender la configuración de las identidades de estos grupos.

Como todos los grupos yumanos, los pa'ipai se clasifican como sociedades cazadoras recolectoras que utilizan territorios diferentes entre la sierra y la costa de acuerdo con las variaciones climáticas y estacionales. El territorio pa'ipai comprendió originalmente la planicie del Álamo, Valle de la Trinidad y hasta el Golfo de California, lugar donde limitaba con el territorio cucapá en el bajo Delta del Río Colorado.

Estos grupos han pasado por distintos procesos y etapas que establecieron modificaciones importantes a su identidad, por ejemplo la llegada de los primeros exploradores venidos de Europa en 1533 (León y Magaña, 2006), o la etapa misional donde intervinieron frailes jesuitas (1697-1768), franciscanos (1768-1773) y dominicos (1773-

12 Teresa Castro reside en Agua Escondida, en las afueras de Santa Catarina. A sus 79 ańos se le considera una de las ancianas y de las principales artesanas de la población. Es una de las últimas personas que por más de 50 años ha realizado la recolección del piñón en la sierra de Juárez, primero con sus padres y hermanos, y ahora con su propia familia. Además, Teresa trabajó en distintos lugares con sus padres, desde San Vicente en la costa del Pacífico hasta el Rancho La Mariana en el Valle de Mexicali. Su historia nos permite entender las transformaciones de la identidad entre los yumanos, en el contexto de las transformaciones de las prácticas de seminomadismo y de recolección en el siglo XX. 
1849); así como la etapa posterior donde se formaron los ranchos en esta región entre 1849 a 1935 (Magańa, 1999) y finalmente la creación de los ejidos indígenas en 1935. Desde la formación de los ejidos indígenas, como veremos con los testimonios de las personas entrevistadas, las actividades de recolección y la vida seminómada estacional se transformaron por la búsqueda de actividades económicas en los ranchos de la región (Garduño, 2001), entre otras.

Actualmente, la forma de ver el mundo de los pa'ipai está vinculada, en parte, con ciertas maneras de realizar y de interactuar durante el periodo de recolección del piñón. Las narrativas biográficas compiladas por Cruz (2015) a propósito de esto identifican que las personas de mayor edad asignan gran valor a los sucesos ligados a tal actividad; ${ }^{13}$ la vida para los pa'ipai, según estos relatos, mantiene su significado en tanto se pueda continuar con la recolección estacional del pińón. Durante las temporadas, grupos de distintos lugares tales como de la Huerta, Santa Catarina o del Mayor Cucapá se reúnen en la sierra. Por ello se puede afirmar que el tiempo de recolección —y por ende de abundanciagenera una mayor interacción entre los miembros del grupo pa’ipai con otros de pueblos yumanos que se siguen yendo a la recolección del piñón y a las actividades asociadas con ésta: la fiesta, el consumo de tequila, el baile y el canto. Así, los asistentes establecen entre ellos relaciones emocionales importantes. El mantenimiento de estas prácticas permite identificar un momento clave en la construcción de la identidad pa'ipai y del resto de los grupos yumanos que participan en la recolección del piñón. Se aprecia así que no existe interés en demostrar o instrumentalizar los elementos de la identidad que son reconocidos como "tradición" por grupos no indígenas, que incluyen cantos, cuentos, danzas, artesanía e idioma. En los encuentros en la sierra, lo que cuenta son las interacciones entre ellos, además de la posibilidad de transmisión de saberes y conocimientos acerca del entorno natural, de la vida y de las actividades de recolección en los momentos de compartir entre las familias y entre los grupos reunidos alrededor del fuego a la luz de la luna serrana:

\footnotetext{
${ }^{13}$ En particular esta información la compartieron Teresa Castro (79 años), Adelaida Albáñez (59 años) y Teodora Cuero (93 años).
} 
Mi papá [era] cantante ... cuando estábamos así acampando, cuando íbamos así al campo, él está hablando en cuentos que nos quería enseñar, o no sé, pero nosotros ni hicimos caso, pero hay veces que canta también así junto a la lumbre, nosotros ni hicimos caso haciendo su cuento, y cantaba pues con su sonaja, y mi mamá decía: "y Pedro qué tiene, está loco”, decía, “ya quiero dormir”, decía, pero así era mi papá muy así era, nos quería enseñar pero nosotros no le hacíamos caso, pues, pero hace falta, verdad, pero pues ya ahora me acuerdo pues así, nos quería enseñar muchas cosas.

Testimonios como el de la señora Teresa Castro ejemplifican cómo esta forma de interacción permitía la transmisión de otras prácticas culturales propias - como tener el espacio para contar los relatos tradicionales o cantar- Al entender que estas actividades sólo tienen lugar en estos espacios y momentos específicos, se identifica la estrecha relación que existe con el territorio como espacio para la reproducción de ciertas cosmovisiones. Sin embargo, en el presente y debido al recorte de las superficies de zonas de recolección o su privatización, e incluso por el aumento de la competencia por el aprovechamiento del recurso, los pa'ipai de menor edad tienen menos posibilidad de experimentar las prácticas ligadas a la recolección del piñón, que ha quedado restringida en mayor medida a los territorios dentro de la comunidad.

Las restricciones espaciales para la recolección del piñón se suman a los cambios de movilidad experimentados por las familias yumanas desde la mitad del siglo XX. Antes, familias como las de Teresa Castro caminaban desde el poblado La Mariana, en las inmediaciones del Delta del Río Colorado en el desierto, hasta la costa del Océano Pacífico en San Vicente, cruzando prácticamente la península de Baja California. En la actualidad los recorridos, los objetivos y la forma en que éstos se realizan, han variado completamente. Si bien las migraciones siguen las rutas de trabajo y siguen siendo motivo de movilidad, la posibilidad del intercambio o comercio con el piñón y otros recursos que se recolectan se vincula a contextos de intercambio comercial regulados desde fuera de sus regiones. 
La privatización de los territorios impide la reproducción de las prácticas culturales vinculadas con la recolección, y los más jóvenes de Santa Catarina posiblemente jamás puedan realizar esta práctica que estaba en el centro de la configuración de la identidad como cazadores recolectores seminómadas estacionales.

\section{Conclusiones: procesos de reconstitución y etnización de los pueblos yumanos en torno a la defensa de territorios, recu- peración de lenguas y continuidad de prácticas culturales}

Si pensamos que los procesos anteriormente descritos forman parte de las experiencias del ser yumano contemporáneo, cabe preguntarse hasta qué punto el despojo y las restricciones para continuar las prácticas son también constitutivas de las subjetividades contemporáneas de cucapás y pa'ipais. En los apartados anteriores se aprecia cómo los miembros de estos pueblos se organizan para contrarrestar - o enfrentar- las dinámicas de desposesión y exclusión de diferentes maneras. Entre los cucapá, por ejemplo, se observa un proceso de creación de un sujeto político colectivo — que crea un sentido de cohesión socialcuando al convertirles en pescadores ilegales dentro de su propio territorio se presentan como pueblo indígena contemporáneo. Los pescadores cucapá organizados en una de las sociedades cooperativas han encontrado la fuerza de su resistencia en su autorreconocimiento como pueblo originario del Delta y usan los instrumentos del derecho - en particular los referentes a los derechos de pueblos y comunidades indígenas - como herramientas para evidenciar las maneras en que los procedimientos de las instancias de pesca y medio ambiente, o las instituciones del modelo económico actual y el aprovechamiento industrial de los recursos naturales, afectan sus formas de vida $y$ patrones de organización y de cultura.

En el caso pa'ipai, los soportes materiales que merman la realización de la recolección son: el campamento militar, los cercos, bardas, paredes o cualquier división del terreno de los ranchos y ejidos en la sierra. Incluso la Asamblea de Bienes Comunales afecta la recolección cuando impulsa lógicas de producción de ámbitos económicos externos a las dinámicas propias de los pa'ipai, posicionándose de esta forma como 
un espacio para la eliminación de las configuraciones culturales de los pueblos yumanos.

Como ya se ha identificado en otro espacio, las asambleas de bienes ejidales o de bienes comunales son instancias que se producen desde el Estado, por lo mismo son ambivalentes, de un lado son la manera de hacer llegar los programas del Estado a los pueblos y comunidades, y por el otro, supuestamente son el vehículo para representar a los indígenas frente a éste (Navarro, 2008b). Lo que aquí observamos es que los procesos que menoscaban las prácticas de recolección son las interacciones entre los miembros de la comunidad que muchas veces se producen dentro de la Asamblea de Bienes Comunales, ${ }^{14}$ también influyen otros factores como la competencia con recolectores no indígenas, y el establecimiento de tiendas y restaurantes que compran sacos de piñón y venden a granel; así como la diversificación de actividades sociales, culturales y económicas que compiten con la recolección de piñón, por ejemplo: el corte de la Palmilla, la elaboración de artesanía, la implementación de proyectos productivos tales como viveros, proyectos de turismo de naturaleza, los trabajos temporales en el mantenimiento de los caminos - actividades que no son culturalmente significativas sino derivadas de intereses económicos de externos-; y en general otros procesos como la migración, la educación y la presencia cada vez mayor de agentes externos. Ante todo, lo que más incrementa la tendencia a desaparecer esta práctica y el conjunto de representaciones que sustenta es la falta de elementos organizativos específicos que los pueblos tienen para darle continuidad y supervivencia.

En este artículo se ha discutido cómo, mientras los pa'ipai intentan continuar con la recolección del piñón a lo largo de la Sierra de Juárez — sin estar todavía muy organizados para demostrar las diferentes instancias que afectan esta práctica-, los cucapá por su parte se organizan contra las limitaciones existentes, y al menos desde una de las tres cooperativas pesqueras se han constituido en sujetos políticos

14 Actualmente no todos los habitantes de la comunidad tienen voz y voto en la Asamblea de Bienes Comunales, debido a que algunos titulares han fallecido. Por ejemplo, Teresa Castro afirma que aun habiendo nacido en Santa Catarina, ella no tiene voz en la asamblea porque el derecho lo portaba su fallecido esposo. 
que demandan se armonicen las leyes medioambientales con el marco de derechos indígenas nacionales e internacionales reconocidos por el Estado mexicano.

Así, las prácticas de recolección del piñón, pero también la recolección y corte de otros frutos secos, raíces, tallos, flores, o incluso de miel silvestres y la pesca son soportes específicos que mantienen prácticas culturales vivas. Se aprecia cómo las prácticas culturales están estrechamente vinculadas con las identidades étnicas locales, generalizables para todos los pueblos yumanos, pues esta permanencia de prácticas de seminomadismo estacional que incluye estancias de cucapás con kiliwas o kumiais con cucapás, o pa ipais y ko’atls con kumiais, se siguen dando hasta el presente. Por lo tanto, la organización social que soporta estas actividades tiene una importancia fundamental para la actualización de la identidad hacia dentro y hacia fuera de las mismas poblaciones yumanas y ante instancias públicas o privadas. $\mathrm{Y}$ es precisamente en esta dimensión de la interacción de los miembros hacia afuera donde se producen los procesos de creación de un sujeto político colectivo: el pueblo indígena contemporáneo.

Algunas iniciativas para ordenar o restringir las actividades productivas al interior de los grupos yumanos — como las normativas de pesca para los cucapá o la propiedad privada en los bosques de piñón para los pa'ipai - violentan las formas de organización propias de estos pueblos que, como ya explicamos, son la pauta para la reproducción de relaciones sociales específicas al interior de los mismos. Además, como también ya explicamos, desdeñan y menoscaban el reconocimiento de las formas de organización propia, sean los sistemas de autoridad culturales o las formas de organización emergentes pero propias, como el caso de las cooperativas entre los pescadores cucapá. Es por ello que la defensa de territorios y de continuidad de prácticas culturales emerge en el nivel político, precisamente porque revela la constitución de sujetos colectivos políticos: los pueblos yumanos contemporáneos.

Aquí se demuestra que los procesos de etnización de los pueblos yumanos —entendiendo etnización como la formación de un sujeto político colectivo (Restrepo, 2011: 40)— se detonan por las acciones de defensa del territorio o el interés de estos pueblos yumanos por 
continuar sus prácticas de recolección. Además de estar articulados con los procesos regionales anteriormente descritos, también se vinculan con elementos del contexto nacional e internacional. Por ejemplo, a nivel internacional, los textos de derecho indígena han sido muy importantes en las acciones y argumentos que los cucapá usan cuando negocian con las autoridades de pesca o de medio ambiente. A nivel nacional, es en los proyectos de revitalización lingüística desarrollados por el Instituto Nacional de Lenguas Indígenas (Inali) ${ }^{15}$ que los cucapá se han planteado preguntas sobre la importancia de compartir el conocimiento de los mayores de manera contextual y no únicamente para fines de documentación de su lengua.

En esta tríada de elementos donde lengua/cultura se configura como un rasgo étnico identitario del "ser indígena", desde donde localizan los conocimientos-practicados ligados al territorio, es que tanto cucapás, ko'atl, kumiai como pa'ipai enfrentan el contraste de estos mundos propios frente a los límites que se construyen al marcar el territorio con otras lógicas que responden a intereses diferentes de los suyos. En ese exterior, el mundo material no es modelado por el gusto de la recolección del pińón que trae aparejada la convivencia, la fiesta y esa reconexión con los que ya no están entre nosotros, ni con el fuego, menos con los campamentos en donde se crea el espacio para la convivencia y el descanso. El mundo de la sociedad mestiza o "mexicana" y de las instituciones del Estado ha reconfigurado y achicado los territorios yumanos siguiendo otro tipo de lógicas asociadas con la propiedad privada, con el uso de los recursos — palmilla, pescado-

${ }^{15}$ La aparición del Inali dentro del marco institucional estatal mexicano puede ser leída como una consecuencia de la coyuntura de lucha iniciada por el EZLN en 1994. De hecho, los diagnósticos del Inali para identificar el número de lenguas indígenas habladas en el país y el desarrollo de programas para revitalizar y fortalecer a las comunidades de hablantes de lenguas indígenas marca un esfuerzo por subvertir los efectos del largo proceso de asimilación cultural a que fueron sometidos los grupos indígenas en México a través de programas específicos diseñados para su asimilación a partir de la creación del INI en 1934. Ese proceso histórico ejercido mediante un proyecto hegemónico y nacionalista devino en la disminución de la diversidad cultural y lingüística del territorio nacional. Sin embargo, actualmente los pueblos yumanos luchan por revertir la situación involucrándose en proyectos de revitalización cultural y lingüística. 
para la comercialización en grandes mercados, imponiendo nuevas reglas al acceso a esos territorios y al aprovechamiento de los recursos naturales que - desde la perspectiva de los propios grupos indígenastiene un significado cultural muy distinto.

\section{Bibliografía citada}

Álvarez de Williams, Anita, 2004, Primeros pobladores de la Baja California, Centro INAH Baja California, Mexicali.

Atlas de Riesgos, elaborado por el gobierno de Baja California, [en línea] disponible en http://www.mexicali.gob.mx/transparencia/ administracion/atlas/pdf/2.pdf [fecha de consulta: 18 de junio de 2015].

Carlsen, Laura, 2014, "Mujeres indígenas denuncian doble discurso de Peńa Nieto en la ONU”, [en línea] disponible en http:// www.cipamericas.org/es/archives/12979 [fecha de consulta: 18 de junio de 2015].

Cruz Hernández, Sergio, 2015, Los recolectores yumanos del piñón: Identidades y representaciones de la relación con la naturaleza en una práctica transgeneracional interpretada desde la perspectiva simbólica, Tesis de doctorado en Ciencias y Humanidades para el Desarrollo Interdisciplinario de la Universidad Autónoma de Coahuila, Centro de Estudios Interdisciplinarios en Ciencias y Humanidades de la UNAM.

Gálvez, Damián, 2013, Acción Política, Poder Local y la Judicialización de Proyectos de Desarrollo en Territorios Indigenas. Un estudio de caso en la Comunidad Mapuche Huilliche Antulafken de Huentetique, Chiloé, Tesis de licenciatura en Antropología, Santiago de Chile, Universidad Academia de Humanismo Cristiano, en http://es.scribd.com/doc/218809014/Galvez-2013-Accionpolitica-poder-local-y-judicializacion-de-proyectos-de-desarrollo-en-territorios-indigenas

Garduño, Everardo, 2001, "De comunidades inventadas a comunidades invisibles: hacia un marco teórico para el estudio de los yumanos de Baja California”, Revista de Estudios Fronterizos, vol. 2, núm. 4, pp. 19-48. 
Garduño, Everardo, 2009, "From invented to imagined and invisible communities: mobility, social networks and ethnicity among the yuman of Baja California", Instituto de Investigaciones Culturales Museo UABC, Mexicali, Baja California, México.

Garduño, Everardo, 2010, "Los grupos yumanos de Baja California: ¿indios de paz o indios de guerra? Una aproximación desde la teoría de la resistencia pasiva”, Estudios fronterizos, vol. 11, núm. 22, pp. 185-205, [en línea] disponible en http:// www.scielo.org.mx/scielo.php?script=sci_arttext\&pid=S018769612010000200007\&lng=es\&tlng=es [fecha de consulta: 05 de agosto de 2014].

Garduño, Everardo, 2013, "Los Pa ipai”, Diccionario Enciclopédico de Baja California, Instituto de Cultura de Baja California, Mexicali.

Gifford, E. W, 1933, “The Cocopah”, University of California Publications in American Archaeology and Ethnology, University of California Press, vol. 31, núm. 5.

Gómez Estrada, José Alfredo, 2000, La gente del delta del Río Colorado: indigenas, colonizadores y ejidatarios, Universidad Autónoma de Baja California, Mexicali, México.

Hohental, W., 2001, Tipai ethnographic notes: a Baja California Indian community at mid-century, Ballena Press, Novato, California, USA.

Inegi, Censo de Población y Vivienda, 2010, [en línea] disponible en http://www.inegi.org.mx/sistemas/olap/proyectos/bd/consulta.asp? $\mathrm{p}=17118$ [fecha de consulta: 18 de junio de 2015].

Kelly, W. H, 1973, Cocopah Ethnography, University of Arizona Press. León, L., \& Magańa, M., 2006, "El periodo misional 1697-1849”, en M. Samaniego, Breve historia de Baja California, pp. 27-62, UABC/Porrúa, Mexicali, Baja California, México.

Leyes Secundarias de la Reforma Energética, [en línea] disponible en http://www.energia.gob.mx/webSener/leyes_Secundarias/ 9220.html [fecha de consulta: 18 de junio de 2015].

López, Julieta y Ovalle, Paola, 2011, "Censo de casas habitadas en Santa Catarina", reporte sin publicar. 
Moseley, Christopher (editor), 2010, Atlas de las Lenguas del Mundo en Peligro, 3ra. edición, Ediciones Unesco, París, disponible en línea en http://www.unesco.org/culture/en/endangeredlanguages/atlas

Montaño, Max., 2015, Ser cucapá: experiencias del ser indígena en Mexicali,Tesis de maestría en Estudios Socioculturales de la Universidad Autónoma de Baja California, Mexicali, B.C., México.

Muelhmann, Shaylih, 2013, Where the river ends. Contested Indigeneity in the Mexican Colorado Delta, Duke University Press.

Navarro Smith, Alejandra, 2008a, "Cucapás, derechos indígenas y pesca. Dilemas del sistema productivo pesquero vis a vis las políticas de conservación de las especies en el Golfo de California”, Revista Chilena de Antropología Visual, vol. 12, núm. 2, pp. 172-196, ISSN 0717-876X, disponible en línea en http://www.rchav.cl/imagenes 12/imprimir/navarro_imp.pdf

Navarro Smith, Alejandra, 2008b, “QQué esperanza que alguien dijera, 'yo soy priísta'! Estado, liderazgos indígenas y faccionalismo político en San_Jerónimo Tulijâ”, Desacatos, núm. 26, enero-abril, pp. 93-118, ISSN: 1405-9274, disponible en línea en http:// www.ciesas.edu.mx/Desacatos/Ini.html

Navarro Smith, Alejandra, 2010, "Cucapás y reconocimiento de sus derechos como pueblo indígena”, en Alejandra Navarro y Carlos Vélez-Ibáñez (coordinadores), Racismo, exclusión, xenofobia y diversidad cultural en la frontera México-Estados Unidos, pp. 87117, Universidad Autónoma de Baja California/Arizona State University, México, disponible en línea en http://issuu.com/ iicmuseo/docs/racismo-exclusion-xenofobia-diversidadcultural $? \mathrm{e}=4384898 / 3596145$

Navarro Smith, Alejandra, 2012, "Representación y antropología visual: videos y construcción de significados sobre lo cucapá", Revista Chilena de Antropología Visual, vol. 2, núm. 20, pp. 79105, Santiago de Chile.

Navarro Smith, Alejandra, 2014, "Reconocimiento de derechos diferenciados en la cultura jurídica central mexicana. 
Apuntes desde el caso cucapá”, Diario de Campo, Tercera época, año 1, núms. 4-5, pp. 15-24, septiembre-diciembre, ISBN $6029340 \quad 033718 \quad 05, \quad$ SEP/Conaculta/INAH, México, disponible en línea en https://docs.google.com/file/ d/0B6EjGmlfyiRyU2poeDVYUl85aFE/edit

Navarro y Bravo, 2015, "Análisis del proceso de consulta para el caso de la pesca cucapá, en Baja California". Ponencia presentada en la mesa Entre la justiciabilidad y la judicialización del derecho indígena en América Latina: Consulta previa, peritajes antropológicos y amparos, en LASA 2015, San Juan Puerto Rico, de 27 a 30 de mayo, manuscrito.

Navarro Smith, Alejandra, Yacotzin Bravo Espinosa, y Catalina LópezSagástegui, 2013, "Legislación de pesca y obstáculos para el reconocimiento de derechos al uso preferencial de recursos naturales del pueblo cucapá", Revista De Estudos e Pesquisas Sobre as Américas, vol. 7, núm. 2., pp. 135-173, ISSN: 19841639, disponible en línea en http://periodicos.bce.unb.br/ index.php/repam/article/view/10026

Navarro Smith, Alejandra, Yacotzin Bravo Espinosa, y Catalina LópezSagástegui, 2014, "Derechos colectivos y consulta previa: territorio cucapá y recursos pesqueros en Baja California", Revista Colombiana de Sociología, vol. 37, núm. 2, pp. 43-64, México, disponible en línea en http://www.revistas.unal.edu.co/index. $\mathrm{php} / \mathrm{recs} /$ article/view/51697/51538

Ortega Esquinca, Agustín, 2004, La comunidad cucapá. Un proceso de formación social en la cuenca baja del Colorado-Gila, Departamento de Prehistoria y Arqueología, Facultad de Geografía e Historia, Universidad de Sevilla, Sevilla.

Porcayo, A. y A. Guía, 2007, “ANEXO 1-Conchas, concheros y las aguas del Golfo de California: análisis arqueozoológico de restos biológicos del área de Mexicali”, Proyecto Registro y rescate de sitios arqueológicos de Baja California fase Municipio de Mexicali, Informe Técnico parcial de la primera temporada y propuesta para trabajo de campo, Centro INAH Baja California, Mexicali. 
Porcayo, A. y A. Guía, 2008, “ANEXO 1-Informe preliminar del análisis de restos arqueofaunísticos del área de Mexicali", Informe de la segunda temporada de campo del Proyecto Registro y rescate de sitios arqueológicos de Baja California fase Municipio de Mexicali y propuesta para la tercera temporada de campo, Centro INAH Baja California, México.

Porcayo, Antonio, Alejandra Navarro, Andrea Guía y Alberto Tapia, 2015, "Cambios y continuidades de la vida ancestral cucapá. Datos arqueológicos, arqueofaunísticos y etnográficos para su comprensión”, INAH, México, en prensa.

Porcayo, Antonio y Juan Martín Rojas, 2009, "Informe de la Tercera Temporada de Campo del Proyecto Registro y Rescate de Sitios Arqueológicos de Baja California Fase Municipio de Mexicali”, Centro INAH Baja California, México.

Porcayo, Antonio y Juan Martín Rojas, 2010, Informe de la Cuarta Temporada de Campo del Proyecto Registro y Rescate de Sitios Arqueológicos de Baja California Fase Municipio de Mexicali. Centro INAH Baja California, México.

Restrepo, Eduardo, 2011, "Etnización y multiculturalismo en el bajo Atrato", Revista Colombiana de Antropología, julio-diciembre, pp. 37-68.

Sieder, R., L. Schjolden y A. Angell (coordinadores), 2011, La judicialización de la politica en América Latina, CIESAS/Universidad Externado de Colombia, México.

Vázquez, Alejandro y Adriana Terven, 2015, "La reinvención del indigenismo. Modernidad, colonialidad y pueblos indígenas", en Alejandro Vázquez (coordinadores), Las geometrías de la desigualdad. Modernidad, globalidad y poder, Universidad Autónoma de Querétaro, Querétaro. 